\title{
Review on Fault Tolerance in Wireless Sensor Network
}

\author{
Ashutosh Choudhary \\ Assistant Professor \\ ASET, AUC
}

\author{
Suniti Choudhary \\ Assistant Professor \\ ASET, AUC
}

\author{
Ankit Mishra \\ Assistant Professor \\ ASET, AUC
}

\begin{abstract}
Data aggregation, information trust, and fault tolerance is considered to enhance the correctness and trustworthiness of collected information. It is based on the multilayer aggregation architecture of WMSNs, existing trust- based framework for data aggregation with fault tolerance with a goal to reduce the impact of erroneous data and provide measurable trustworthiness for aggregated result. DAG scheme offers fault lenience for packet loss by forming a Directed Acyclic Graph (DAG), which allows a node to have multiple parent nodes. This method can ensure correct data transmission timing, according to the actual hop count of the edge of the DAG.
\end{abstract}

\section{Keywords}

Data aggregation, fault tolerance, trustworthiness, Wireless multimedia sensor networks.

\section{INTRODUCTION}

Wireless sensor networks (WSN) require relatable and reliable data collection outlines to provide information about their deployment area. The availability of micro-sensors and low-power wireless communications will enable the disposition of sensor networks for a wide range of new services to support user activities [1]. These networks are likely to be serene of many distributed sensor nodes, using unpredictable wireless links by default, equipping sensors (e.g. light, sound and motion), functioning selfconfigurable, and in many cases, without access to renewable energy resources. Thus, energy saving is one of the relevant issues for achieving long-lived networks [2]. There is another major issue, which is the enhancement in the accuracy of the collected data [3], since obtaining accurate data is the prime objective of the observer. This signifies a challenge for sensor networks to improve their

ability to match the actual value of the extent being collected.

\section{RELATED WORK}

\subsection{Fault Tolerance for event detection in wireless sensor network}

It proposes a new approach that considers the case where nodes can have different failure probability values. Various types of failures can be handled Which can be used as a general distributed fault-tolerance mechanism for any application where nodes may have diverse accuracy levels [3]. Two new dispersed error models that take into account the location and relative position of sensor nodes are developed. The first model takes the fact that nodes that are nearer to each other have a higher spatial correlation than far nodes. The second model accounts for the importance of the relative geographical distributions of the two voting quorums (the two subsets of neighbors deciding the presence of an event or its absence, respectively). It comes from the comment that an event detected on all sides of a node (the node is in the middle of the detection region) is more likely to be present at the node itself than an event that was detected by nodes only on one side of the node (the node might be on the edge of the eventregion).

Several assessment mechanisms that can be used by sensor nodes to learn their error rates continuously are developed [4][5]. The proposed mechanisms utilize the moving average and geometric moving average algorithms. These two schemes can provide accurate and timely estimation of the node error rate when their parameters are set. No longer Nodes required to know their error rates prior to the deployment. It allows the algorithm to handle the situation where the node error rate changes over time.

\subsection{Fault Tolerance in Spatial Query Processing}

The core involvement of this paper is the intention of an energy efficient fault-tolerant mechanism for in-network spatial query handling in WSN. Query propagation avoids failed nodes by requesting applicants to forward the query, thus considering only the nodes that responded to the request. This approach increases the robustness and energy efficiency of the mechanism, since there is no need of node monitoring for failure detection. The mechanism is composed of three new algorithms. The first is a fault tolerant geographic routing protocol to forward queries to the region of interest (RoI). It also proposes two failure resilient algorithms to disseminate queries within the RoI, collect environmental data and aggregate them.

There are two types of spatial queries in WSN: window and KNN. In window queries, the user defines the RoI inside the area monitored by a WSN and nodes inside this region collect environmental data [6]. Another type of query is called KNN (K Nearest Neighbor). The user defines a point inside the monitored area (called query point) and the value of $\mathrm{K}$. The data is collected by the $\mathrm{K}$ nodes contiguous to the query point [7]. In this work we focus on window query processing.

Spatial query processing can be divided into six stages, as illustrated by Figure 1 . The user, represented by the computer, defines the RoI. The Pre-Processing stage is performed in the user's computer. It prepares queries to be sent to the WSN. The first sensor node to receive the query is named Originator. In the Forwarding stage, the query is forwarded from the Originator to a node within the RoI. This last node in the Forwarding stage is called Coordinator. In the Dissemination stage, the query is disseminated from the Coordinator to all nodes inside the region of interest. These nodes sense environmental data in the Sensing stage and send this information to be aggregated in the Aggregation stage. Finally, in the Return stage, the node with the query result forwards it to the Originator. 


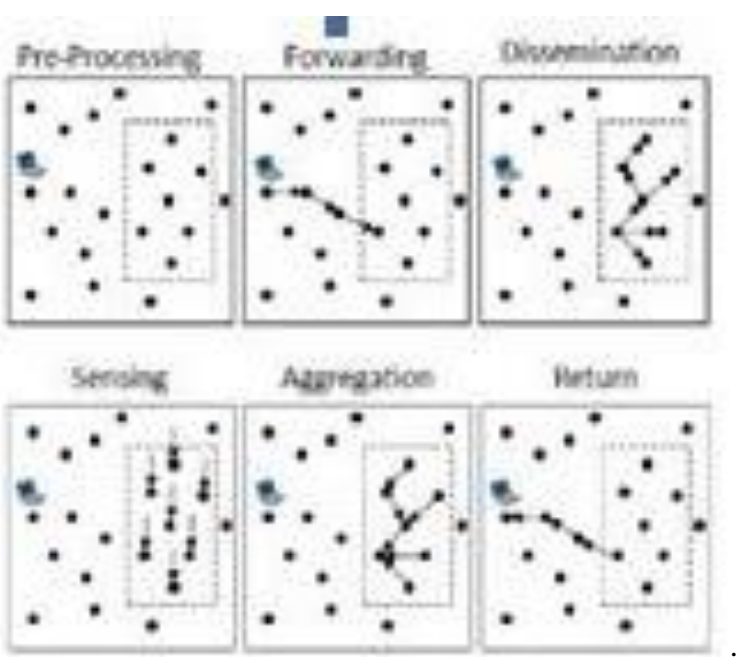

Fig1: Six Stages of Spatial Query Processing

\subsection{Layer Independent Fault Tolerant for wireless sensor network}

It presented a layer independent fault tolerance mechanism which applies to critical monitoring applications. It allows monitoring reports to be stored during failures before it's finally retransmitted from false data sources. It preserved a stateless mechanism, which ensured a complexity of low message and a low energy consumption. A repair-based solution therefore leads to a fast recovery once new paths open toward sink stations [8].

The main objective of LIFT consists in the storage of monitoring reports whenever every next hop relay to the sink becomes unavailable. Considering a mobile environment (e.g. inter-vehicle communication), such situation may occur whenever nodes located on the routing path become out of range resulting in a network partition. Its aim at benefiting from both the in-node resources and the ones located in its vicinity. It then proposes to have stored nodes acting as phony data sources once next hops get available again. Upon such recovery, stored packets are delivered to the sink in order to achieve reliable data collection, without emerging from an overt fault-tolerance mechanism [9]. These messages are indeed measured as standard monitoring reports. All operations defined in LIFT are shown in Figure 2.

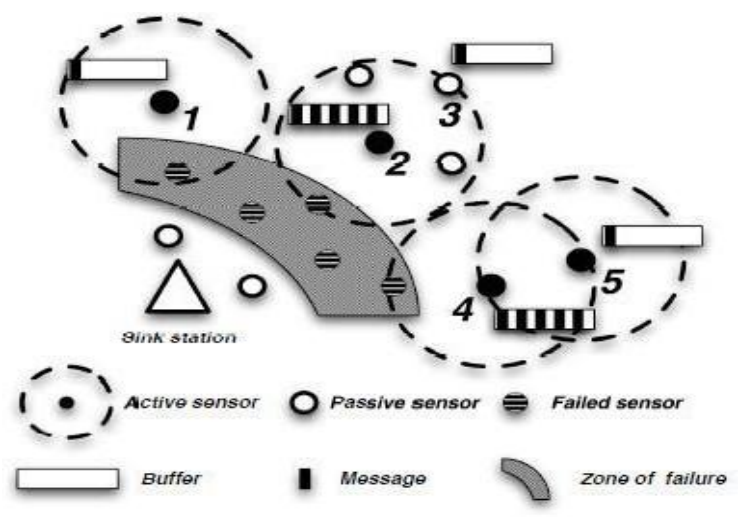

Fig2: Operation of LIFT

\subsection{Fault Tolerant Routing Techniques in Wireless Sensor networks}

Fault tolerance confirms that a system is available for use without any disruption in the incidence of faults; thus fault tolerance rises the reliability, availability, and consequently dependability of the system. The most popular approach for fault tolerance is the multipath routing where a set of several paths between the source nodes and the sink are determined at the payment of increased energy ingestion and traffic generation. Multipath routing provides additional benefits of load balancing and bandwidth aggregation. Routing protocols proposed for WSNs [14, 15] can be classified into three groups depending on the methods used for finding the path, proactive routing in which all paths are computed and maintained in advance and stored in a routing table, reactive routing where all paths are generated on demand, and hybrid routing which is a mix of the both the groups.

Two mechanisms [16] are used to establish multiple paths. Disjoint multipath build a number of alternate paths which are node/link disjoint with the primary path and with other alternate paths. Thus, a failure in any or all nodes/link on the primary path does not affect the alternate paths. Those alternate paths expend meaningfully more energy than that on the primary path since they could potentially have much longer latency; moreover, global topology knowledge is needed to facilitate the creation of the multiple disjoint paths. Using this multipath scheme in a network with $k$ node-disjoint routes from source to destination can tolerate at least $k-1$ intermediate network factor failures. Braided multipath construct an alternative path for each node in the primary path that does not include this node, which means alternate paths in a braid partially overlay with the primary path. These alternate paths are not much more expensive than the primary path in terms of latency and overhead (alternative paths are shorter than in disjoint multipath). However, when all or most of the nodes on the primary path fail, new path discovery is required which introduces a surplus overhead.Fault tolerance mechanisms are usually classified into retransmission and replication types.

\section{Retransmission}

The most popular mechanism is to retransmit the data packets to the sink on one of the multiple paths using minimum hop count or minimum energy ingestion depending on the network requirement, for a predetermined number of times. The way is that the sink node transfers an acknowledgement back to the basis when a data packet is received indicating successful transmission. If the acknowledgement is not received by the sender before a timeout, the data packet will be retransmitted. In WSNs the packet loss rate on the wireless link is higher than in other networks, thus the link level retransmissions is the most popular mechanism used. However, this method has some drawbacks in that it increases the network traffic requiring more resource consumption. Additionally, transmitting an acknowledgment message may increase delivery delay and more packet loss due to collisions. Furthermore, more memory space is needed in the sensor nodes to buffer the packet until it receives an acknowledgment from the destination.

\section{Replication}

Introducing redundancy into packet delivery [17] is another mechanism used to provide fault tolerant routing protocols for WSNs. One of the replication mechanisms that routing protocols adopt to ensure delivery of the original packet to the sink is to transmit multiple copies of the same packetover different paths in order to recover from some path failures. The major drawbacks of this mechanism are the high overhead introduced when the packet 
is transmitted through each node till it reaches the sink, the maintenance of the path state in each of these nodes, and not being adaptive to channel errors.

\subsection{Outlier Detection Based Fault Tolerant Data Aggregation for Wireless Sensor Networks}

A. Error lenient Data Aggregation scheme using an innetwork outlier detection mechanism, called FTDA. The outlier detection mechanism is based on the Locality Sensitive Hashing (LSH) technique [18]. The LSH algorithm used in FTDA permits solid representation of sensor data which reduces the communication overhead of outlier detection. FTDA takes advantage of LSH technique by estimating the similarity of sensor data from their compact sketches (LSH codes). The data aggregator performs data aggregation based on these LSH codes.

First, we propose a novel fault tolerant data aggregation scheme, FTDA, using an in-network outlier detection mechanism based on LSH technique. With the help of LSH Practice, FTDA protocol is able to detect outliers in a distributed and energy efficient manner. Second, using LSH codes, FTDA protocol eliminates the redundant data transmission from sensor nodes to data aggregators thereby incrementing the efficiency of data aggregation process.

FTDA protocol involves of three phases,

(i) Data collection and LSH code generation,

(ii) Outlier detection and redundant data elimination, (iii) Data aggregation.

These three phases are periodically realized in each cluster. This paper presents a fault tolerant data aggregation scheme that eliminates the false data sent by malfunctioning and/or compromised sensor nodes. To prolong the lifetime of the network by saving energy, a Locality Sensitive Hashing (LSH) based in-network outlier detection technique is used. The simulation results display that the planned scheme, FTDA, is able to detect outliers in most cases. As a result, FTDA reduces the number of false data transmissions thereby increasing the data aggregation accuracy.

\subsection{F. A Trust-Based Framework for Fault-Tolerant Data Aggregation in Wireless Multimedi}

a Sensor Networks

In WMSNs, data aggregation with fault-tolerance can reduce the network load and guarantee data reliability. As WMSNs are associated with different applications which have different data types, data fault-tolerance will vary from applications to applications. Most of the fault-tolerant techniques [19], [20] make use of temporal or spatial correlation, and statistical characteristics to reduce the impact of erroneous data. During the process of data gathering and aggregation, each node calculates its selftrust opinion on its collected data through temporal correlation, and then sends the data along with its self-trust opinion to its aggregator.

Each aggregator estimates its peer-trust opinion on each node in its aggregation set through spatial correlation, and then calculates its peer-trust opinion on the data reported by each child node using trust transfer rule. Based on the trust opinions calculated, each aggregator performs weighted data aggregation, and derives self-trust opinion on its aggregated result using trust combination rule.
Subsequently, each aggregator reports the aggregated result and its self-trust opinion to the upper layer aggregator progressively, till the sink node. The proposed scheme can significantly decrease the impact of erroneous data and provide measurable trustworthiness for aggregated data

\section{CONCLUSIONS}

The proposed method forms a Directed Acyclic Graph (DAG), that allows a node to have multiple parents, and collects data using the DAG. By using multiple parents as intermediate nodes, which provides tolerance for the failure in wireless transmission. Although the method uses multiple parents, the method avoids being aggregated by the same. In addition, the method can tune the data transmission timing control correctly according to the actual hop count of edge of the DAG.

By using combination of two methods:

(1) Trust based method

(2) DAG method

\section{REFERENCES}

[1] Prof. Rakhi Bhardwaj, Prof.Sandesh S.Jangam, Prof.Prashant.N.Shinde, Prof.Abhijit.B.Raut, Prof.RajeshS.Trigune,"LAN Monitoring using Android Phone", International Journal of Innovative Research in Computer and Communication Engineering(An ISO 3297:2007 Certified Organization,Vol.2,Issue 2,February,2014)

[2] Prof Harsha Thadani, Prof. Supriya Kumari, Prof. Miranuddin Shaikh, Prof. Neha Baravkar, Prof. Shubhangi Kale,"Monitoring PCs using Android",International Journal of Scientific \& Engineering Research(ISSN 2229-5518 Volume 4, Issue 4, April-2013 1537)

[3] Prof.Pooja Chaudhary, Prof. Manasi Bhutada, Prof.Atul Bavoria, "WLAN Monitoring Using Android Phone",IJREAT International Journal of Research in Engineering And Advanced Technology,(ISSN: 2320 - 8791 Impact Factor: 1.479, Volume 2, Issue 3, June-July, 2014)

[4] Prof. Gowthami.T, Dr. Adiline macriga. G, "Smart Home Monitoring and Controlling System Using Android Phone", International Journal of Emerging Technology and Advanced Engineering,(ISSN 2250-2459, ISO 9001:2008 Certified Journal, Volume 3, Issue 11, November 2013)

[5] Prof.Dhanke D.T., Prof.Bodkhe S.S., Prof.HambardeS.M., Prof.Vaidya R.P., "LAN Monitoring and Controlling using Android", International Journal of Advanced Research in Computer Engineering \& Technology,(Volume 3 Issue 3, March 2014)

[6] Prof. Mamata Bhamare, Tejashree Malshikare, Renuka Salunke, Priyanka Waghmare, "GSM Based LAN Monitoring and Controlling", InternationalJournal of Modern Engineering Research,(IJMER)(ISSN:2249-6645,www.ijmer.com Vol.2, Issue.2, Mar-Apr 2012 pp-387-389)

[7] Amol Poman, Mahesh Gundras, Prashant Pujari, "GSM Based LAN Monitoring System", Amol Poman et al,/(IJCSIT) International Journal of Computer Science and Information Technologies, ( Vol. 3 (3) , 2012,3848-3851)

[8] Prof. C. S. Nimodia, Prof. S. S. Asole, "A Survey On Network Monitoring \& Aministration Using Email \& Android Phone"International Journal of Emerging Technology and Advanced Engineering ,Website: www.ijetae.com (ISSN 22502459, ISO 9001:2008 Certified Journal, Volume 3, Issue 4, April 2013) 
[9] Sonam Gavhane, Rasika Phanse,B.W.Balkhande,"Remote Desktop on Mobile" International Journal of Innovations in Engineering and Technology (IJIET)(Vol. 2 Issue ISSN:2319-1058,February 2013)

[10] Ashutosh Kumar Choudhary,Piyush K.Ingole "Smart phone based approach to monitor driving behaviour and sharing of statistic. 2014 Fourth International Conference on Communication Systems and Network Technologies 978-1-4799-3070-8/14 \$31.00 (c) 2014 IEEE DOI 10.1109/CSNT.2014.61.
[11] Ashutosh Kumar Choudhary," Study of Various Routing Protocols in VANET" International Journal of Emerging Trends in Engineering and Development, Issue 8, Vol.1 (January 2018) DOIhttps://dx.doi.org/10.26808/rs.ed.i8v1.02

[12] Suniti Purbey." A REVIEW ON MANET (MOBILE AD HOC NETWORK)" International Journal of Emerging Trends in Engineering and Development Issue 9, Vol.1 (January 2019) DOI https://dx.doi.org/10.26808/rs.ed.i9v1.02 